\title{
Decreased expression of ADAMTS-1 in human breast tumors stimulates migration and invasion
}

\author{
Vanessa M Freitas ${ }^{1 *}$, Jônatas Bussador do Amaral ${ }^{1}$, Thaiomara A Silva ${ }^{1}$, Emerson S Santos ${ }^{1,2}$, Flávia R Mangone ${ }^{3}$, \\ João de Jesus Pinheiro ${ }^{1,4}$, Ruy G Jaeger ${ }^{1}$, Maria A Nagai ${ }^{3}$ and Gláucia Maria Machado-Santelli ${ }^{1}$
}

\begin{abstract}
Background: ADAMTS-1 (a disintegrin and metalloprotease with thrombospondin motifs) is a member of the ADAMTS family of metalloproteases. Here, we investigated mRNA and protein levels of ADAMTS-1 in normal and neoplastic tissues using $\mathrm{PPCR}$, immunohistochemistry and immunoblot analyses, and we addressed the role of ADAMTS-1 in regulating migration, invasion and invadopodia formation in breast tumor cell lines.
\end{abstract}

Results: In a series of primary breast tumors, we observed variable levels of ADAMTS-1 mRNA expression but lower levels of ADAMTS-1 protein expression in human breast cancers as compared to normal tissue, with a striking decrease observed in high-malignancy cases (triple-negative for estrogen, progesterone and Her-2). This result prompted us to analyze the effect of ADAMTS-1 knockdown in breast cancer cells in vitro. MDA-MB-231 cells with depleted ADAMTS-1 expression demonstrated increased migration, invasion and invadopodia formation. The regulatory mechanisms underlying the effects of ADAMTS-1 may be related to VEGF, a growth factor involved in migration and invasion. MDA-MB-231 cells with depleted ADAMTS-1 showed increased VEGF concentrations in conditioned medium capable of inducing human endothelial cells (HUVEC) tubulogenesis. Furthermore, expression of the VEGF receptor (VEGFR2) was increased in MDA-MB-231 cells as compared to MCF7 cells. To further determine the relationship between ADAMTS-1 and VEGF regulating breast cancer cells, MDA-MB-231 cells with reduced expression of ADAMTS-1 were pretreated with a function-blocking antibody against VEGF and then tested in migration and invasion assays; both were partially rescued to control levels.

Conclusions: ADAMTS-1 expression was decreased in human breast tumors, and ADAMTS-1 knockdown stimulated migration, invasion and invadopodia formation in breast cancer cells in vitro. Therefore, this series of experiments suggests that VEGF is involved in the effects mediated by ADAMTS-1 in breast cancer cells.

Keywords: ADAMTS-1, Breast cancer, Migration, Invasion, VEGF

\section{Background}

Extracellular matrix (ECM) components are involved in various aspects of tumor biology, including metastatic events. The ECM provides solid support to cells as well as a supply of cytokines and growth factors [1,2]. Cancer progression depends not only on the new abilities gained by neoplastic cells but also on the interaction between cells and their microenvironment [3]. ECM components are cleaved by proteases during physiological and pathological processes, and protease-induced breakdown of the

\footnotetext{
*Correspondence: vfreitas@usp.br

'Departamento de Biologia Celular e do Desenvolvimento, Instituto de Ciências Biomédicas, Universidade de São Paulo, Av. Prof. Lineu Prestes 1524, Ed Biomédicas 1 sala 428, São Paulo, SP 05508-000, Brazil

Full list of author information is available at the end of the article
}

ECM is essential for cancer cells to move through tissue barriers [4].

ADAMTS-1 (a disintegrin and metalloprotease with thrombospondin motifs) is a member of the ADAMTS family of metalloproteases. This secreted protease participates in various biological processes, such as inflammation, angiogenesis and development of urogenital system [5-8]. ADAMTS-1 has specific substrates that include modular proteoglycans, such as versican, aggrecan and brevican [9]. Despite the suggested roles for ADAMTS-1 in tumor invasion and metastasis, the effects of this molecule during cancer progression remain controversial. In 2008, Rocks et al. [10] showed that ADAMTS-1 contributes to tumor development by attracting fibroblasts and remodeling the extracellular matrix. Furthermore, some authors 
have shown that ADAMTS-1 is upregulated in pancreatic cancers with metastatic phenotypes [11]. On the other hand, decreased ADAMTS-1 expression has been described in human malignancies [12]. As a result, this protease was initially thought to inhibit angiogenesis in cancer and therefore act as an anti-cancer molecule [6] via the blockade of VEGFR2 phosphorylation by directly binding and sequestrating VEGF165 [7].

Here, we analyzed ADAMTS-1 mRNA expression in 60 human breast tumors, protein localization in 59 human samples and protein expression in 56 human samples, including normal and neoplastic tissues. To further evaluate the role of ADAMTS-1 in tumor biology, we studied the role of this protease in the regulation of migration and invasion in MDA-MB-231 and MCF7 breast cancer cells. Knocking down ADAMTS-1 expression using siRNA increased the migration and invasion of MDA-MB-231 cells. We also found a relationship between ADAMTS-1 and the activity of invadopodia, membrane protrusions related to the initial steps of cancer invasion. MDA-MB-231 cells with silenced ADAMTS-1 displayed increased VEGF expression in conditioned medium. Furthermore, this conditioned medium containing higher levels of VEGF induced HUVEC tubulogenesis. Our findings indicate that the effects of ADAMTS-1 in tumor invasiveness may be related to the availability of VEGF.

\section{Results}

\section{ADAMTS-1 protein and mRNA expression in primary breast tumors}

Quantitative real-time RT-PCR (qPCR) was used to analyze mRNA expression level of ADAMTS- 1 in 60 primary breast tumors. ADAMTS-1 transcripts were expressed at varying levels (ranging from 0.1 to $7.3 \mathrm{AU}$ ) in the primary breast tumors analyzed. We determined a gene expression cut-off value of 0.7 (median value) that differentiated between ADAMTS-1 low expression and high expression in breast tumors. Using this cut-off, 39/60 (65\%) of the breast tumors were classified as ADAMTS-1 low expressors, and 21/60 (35\%) were classified as ADAMTS-1 high expressors. The clinicopathological characteristics of the patients are summarized in Table 1.

Table 2 compares the mean ADAMTS- 1 mRNA expression values in relation to the clinicopathological features of the 60 breast cancer patients.

Statistical analyses demonstrated that high ADAMTS-1 mRNA expression was significantly associated with advanced clinical stages $(\mathrm{p}=0.006)($ Table 2 and Figure 1$)$. However, there were no significant differences between ADAMTS-1 mRNA expression and age, tumor size, lymph node metastasis or the levels of estrogen or progesterone receptors. Neither overall survival (log-rank test; $\mathrm{p}=0.459$ ), nor disease-free survival (log-rank test; $\mathrm{p}=0.481$ ) were
Table 1 Patients and tumor samples characteristics

\begin{tabular}{lll}
\hline Variable & Categories & $\boldsymbol{n}$ \\
\hline Age (years old) & $<50$ & $22(36.7)$ \\
Stage (TNM) & $\geq 50$ & $38(63.3)$ \\
& Stage I & $2(3.3)$ \\
& Stage II & $25(41.7)$ \\
& Stage III & $26(43.3)$ \\
Tumor size (cm) & Stage IV & $7(11.7)$ \\
Lymph node metastasis & $<4.0$ & $21(35.0)$ \\
Estrogen receptor & $\geq 4.0$ & $39(65.0)$ \\
Progesterone receptor & No & $20(33.3)$ \\
& Yes & $40(66.7)$ \\
& Negative & $27(45.0)$ \\
ADAMTS-1 & Positive & $33(55.0)$ \\
& Negative & $33(55.0)$ \\
& Positive & $26(43.3)$ \\
& Missing cases & $1(1.7)$ \\
& Negative & $31(51.7)$ \\
& Positive & $29(48.3)$ \\
\hline
\end{tabular}

significantly different between patients with low or high ADAMTS-1 mRNA expression levels (data not shown).

Immunoblot analyses using an antibody raised against the N-terminus of ADAMTS-1 produced at least four different bands in samples from human patients, with approximate molecular weights of $110,80,70$ and $60 \mathrm{kDa}$ (Figure 2A). Moreover, the levels of ADAMTS-1 expression

Table 2 ADAMTS- 1 expression (median value) according to clinicopathological features and biomolecular markers in primary breast tumors

\begin{tabular}{lllll}
\hline Variable & Categories & $\boldsymbol{n}$ & $\begin{array}{l}\text { ADAMTS-1 } \\
\text { expression }\end{array}$ & $\boldsymbol{p}$ value \\
\hline Age (years old) & $<50$ & 22 & 0.350 & MW: 0.534 \\
& $\geq 50$ & 38 & 0.475 & \\
Stage (TNM) & Stage I & 2 & 0.225 & KW: 0.006 \\
& Stage II & 25 & 0.250 & \\
& Stage III & 26 & 0.625 & \\
Tumor size (cm) & Stage IV & 7 & 1.100 & MW:0.950 \\
& $<4.0$ & 21 & 0.350 & MW: 0.712 \\
Lymph node metastasis & No & 39 & 0.400 & \\
& Yes & 20 & 0.450 & MW: 0.291 \\
Estrogen receptor & Negative & 27 & 0.350 & MW: 0.731 \\
& Positive & 33 & 0.600 & \\
Progesterone receptor & Negative & 33 & 0.550 & \\
& Positive & 26 & 0.350 & \\
\hline
\end{tabular}

KW: Kruskal Wallis test. MW: Mann Whitney test. 


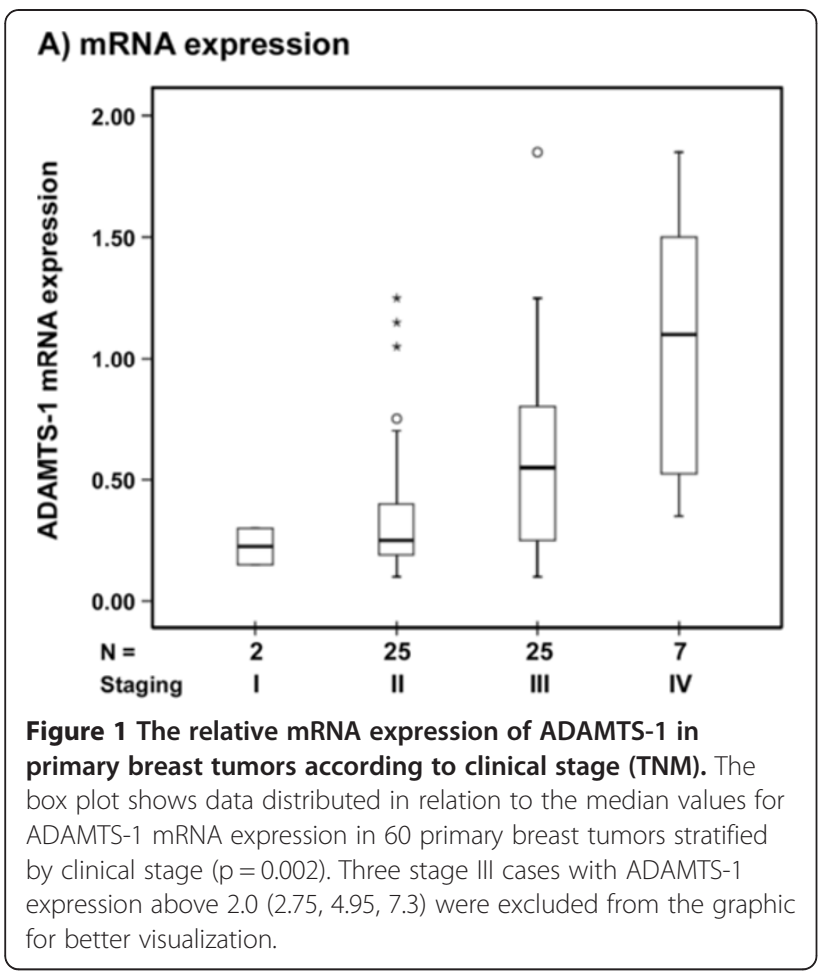

in tumors was lower compared to the expression levels in adjacent, normal tissues from the same patients, and this comparison was obtained by calculating the ratio between ADAMTS-1 and tubulin expression. The $80 \mathrm{kDa}$ ADAMTS-1 form produced the strongest band, and its expression was lower in tumors as compared to normal adjacent tissues (Figure 2B). No differences were found among the other three bands when comparing the tumor samples to normal adjacent tissues (not illustrated).

Immunohistochemistry (IHC) was carried out in samples from normal tissue; breast cancer tumors of grade IIA, IIB, IIIA and IIIC; and metastatic lymph nodes, and we evaluated ADAMTS-1 expression in the tumor cell cytoplasm and the surrounding stroma (Figure 3A). No significant differences were observed regarding the staining area fraction, even in the higher graded tumors (Figure 3B). However, high-grade tumors (IIIA and IIB) exhibited a trend towards decreased ADAMTS-1 expression in comparison to the lower tumor grades.

Next, we compared tumor and normal tissue subgroups, with tumor samples classified as either 1) estrogen (ER) and progesterone (PR) receptor positive tumors or 2) triple-negative tumors (ER-, PR-, Her-2). In triple-negative tumors, ADAMTS-1 protein expression was reduced in $40 \%$ of the cases as compared to both normal tissues and the ER or PR single-positive samples (Figure 3C).

We then assessed differences in staining intensity with regard to the ADAMTS-1 distribution among tumor cells or stroma. The labeling intensity profile (Figure 3D) showed that the expression of ADAMTS-1 in normal tissues was prominent in both epithelial cells and stroma. In contrast, ADAMTS-1 staining was discrete or absent in the tumor stroma, and this discrete or absent staining pattern was more evident in cases with higher grades of malignancy.

\section{Effects of ADAMTS-1 expression on cell migration}

We also investigated ADAMTS-1 expression levels in breast tumor cell lines, such as MCF7 and MDA-MB231. In these cells, siRNA treatment reduced the expression of ADAMTS-1. In addition, cell lysates and conditioned medium were analyzed by immunoblot, and an $80 \mathrm{kDa}$ band was observed for the conditioned medium from both cell lines (Figure 4), whereas a $40 \mathrm{kDa}$ band was present predominantly in the cell lysates. In

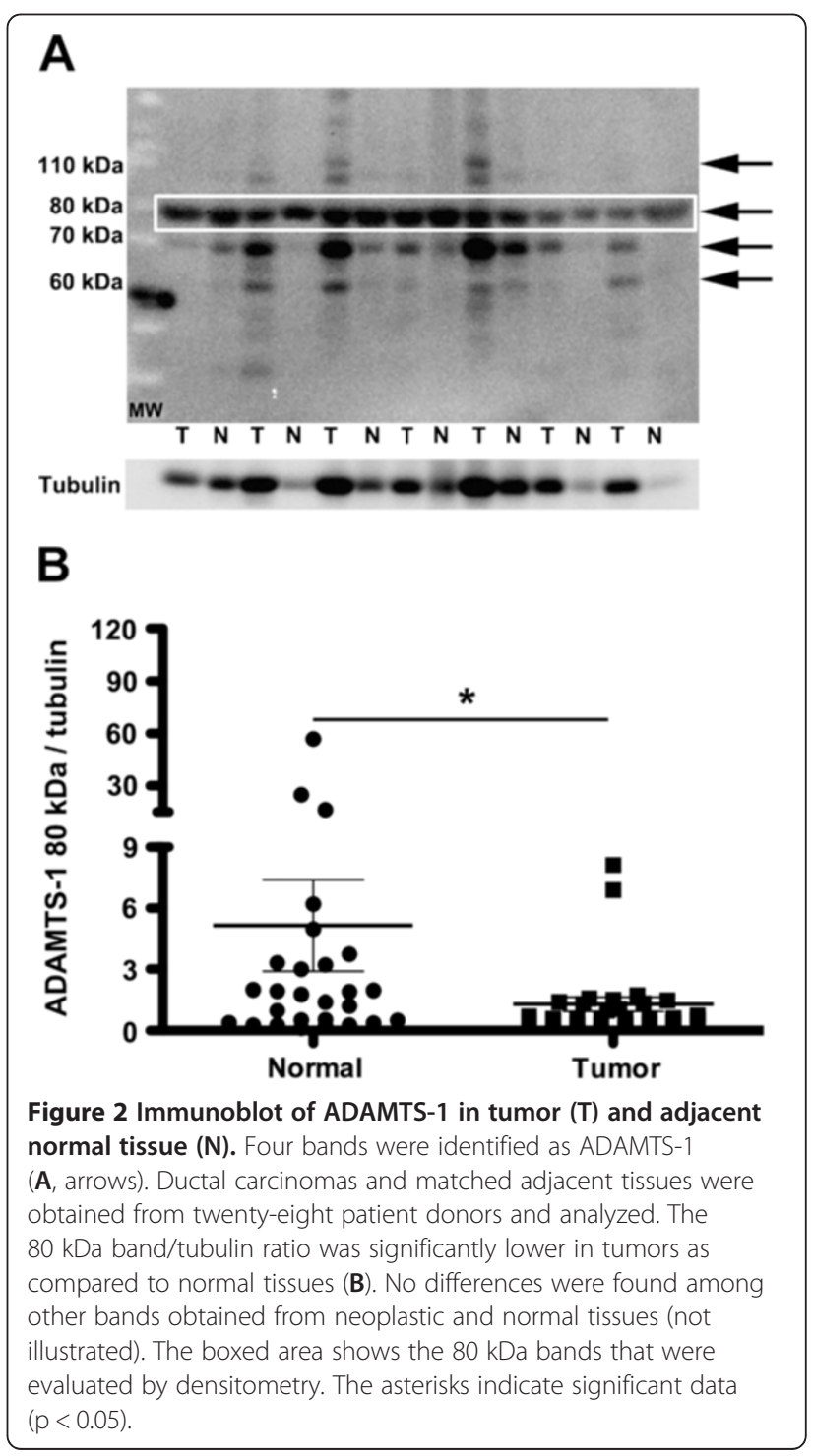




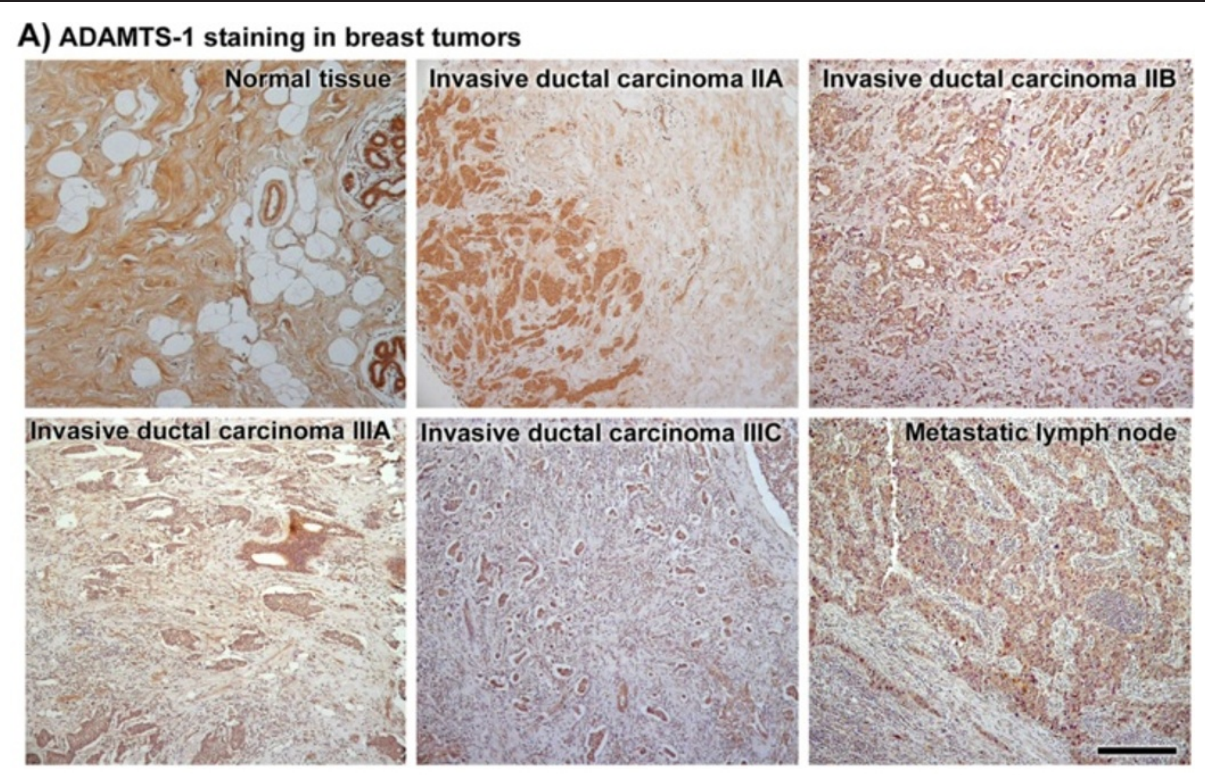

\section{B) ADAMTS-1 Protein levels}

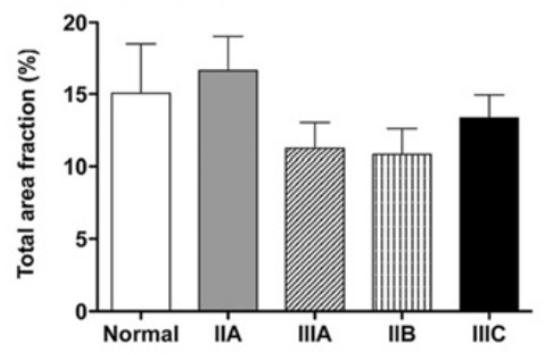

\section{C) ADAMTS-1 subgroup analyses}

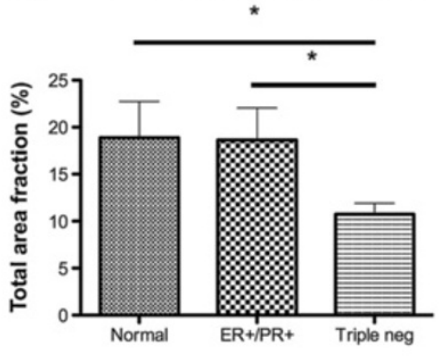

\section{D) ADAMTS-1 intensity profile}
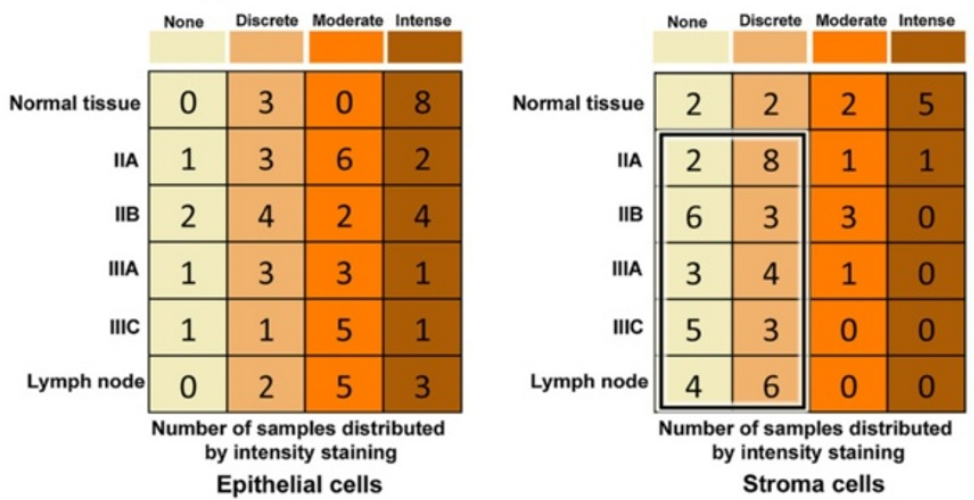

Figure 3 ADAMTS-1 was more abundant in normal tissue stroma as compared to tumor stroma. ADAMTS-1 staining is shown for normal tissue, invasive ductal carcinoma of grades IIA, IIB, IIIA and IIIC and metastatic lymph nodes (A). When the total area fraction was analyzed by immunohistochemistry, ADAMTS-1 showed no statistically significant difference in staining between normal and tumor tissues (B). However, a decreasing trend was observed in high-grade types (IIIA and IIB). Subgroup analysis revealed a significant decrease in ADAMTS-1 expression in triple negative tumors as compared to normal tissues and ER- or PR-positive tumors (C). The labeling intensity profile (D) shows that the expression of ADAMTS-1 in normal tissue was prominent in both epithelial cells and stroma. In contrast, ADAMTS-1 staining was discrete or absent in tumor stroma (D, boxed area), and this discrete or absent staining pattern was more evident in higher grades of malignancy (D, boxed area). Scale bar: $50 \mu \mathrm{m}$. 


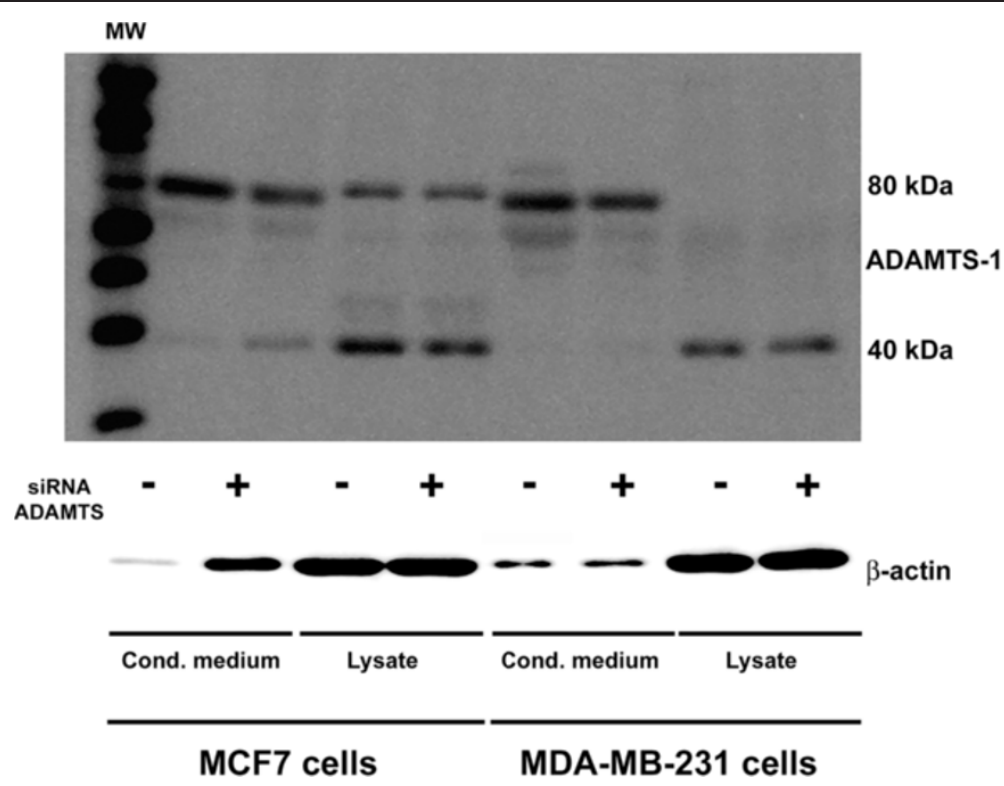

Figure 4 ADAMTS-1 expression in cell lysates and conditioned medium from MCF7 and MDA-MB-231 cells. These cells showed reduced expression of ADAMTS-1 following siRNA treatment. The lysates and conditioned media were analyzed by immunoblot, and an 80-kDa band was prevalent in the conditioned medium from both cells lines, but more prominently in MDA-MB-231. A 40-kDa smaller band was predominant in the cell lysates. The immunoblot also illustrated the efficiency of ADAMTS-1 siRNA knockdown. These experiments were carried out at least six times, and similar results were consistently obtained.

addition, the immunoblot illustrated the efficiency of ADAMTS-1 siRNA knockdown (Figure 4).

Next, the effect of ADAMTS-1 on cell migration was analyzed using time-lapse imaging. For these experiments, we utilized shRNA-GFP. Cells were transfected with either ADAMTS-1 shRNA-GFP or control shRNA-GFP, and only fluorescent cells were analyzed. We measured the cell distance from the initial position during a set time interval, which yielded a mean velocity measurement ( $\mu \mathrm{m} /$ hours). Functional ADAMTS-1 knockdown stimulated the migratory activity of MDA-MB-231 cells, and these cells presented a mean velocity of $11.74 \pm 0.9603 \mu \mathrm{m} /$ hour, which was at least 2 times higher than that of the controls (4.756 $\pm 0.3993 \mu \mathrm{m} /$ hour) (Figure 5A-D).

MCF7 cells were used in migration experiments to address whether ADAMTS-1 knockdown stimulated migration in other cell lines, including this non-invasive breast adenocarcinoma cell line. The mean velocity of the control cells was $6.479 \pm 0.4896 \mu \mathrm{m} /$ hour, whereas the velocity of the treated cells was $4.676 \pm 0.3026 \mu \mathrm{m} /$ hour. Therefore, in MCF7 cells, migration was not stimulated and was instead reduced (Figure 5E-H).

\section{VEGF availability and VEGFR2 expression}

As ADAMTS-1 knockdown had different effects in MCF7 and MDA-MB-231 cells, we next focused on the mechanisms of ADAMTS-1 that were responsible for these effects on cell migration. Previous reports have demonstrated that ADAMTS-1 sequesters VEGF [7]. Thus, we hypothesized that decreasing ADAMTS-1 could increase the availability of VEGF. ELISAs were performed to determine the relative VEGF concentrations in conditioned medium from either MDA-MB-231 or MCF7 cells that received ADAMTS-1 siRNA knockdown treatment. We found that MDA-MB-231 cells in conditioned medium exhibited a statistically significant increase in VEGF when ADAMTS-1 expression was reduced (Figure 6A). Conversely, MCF7 cells in conditioned medium showed a decrease in VEGF when ADAMTS-1 was silenced using siRNA (Figure 6A).

To address the functional role of decreased ADAMTS-1 levels in MDA-MB-231 cells and the consequent increase in VEGF, we carried out endothelial tubulogenesis assays.

In MDA-MB-231 cells, ADAMTS-1 expression was silenced by siRNA, and cells transfected with scrambled siRNA served as the controls. We obtained conditioned medium from treated and control cells, and we used this conditioned medium to induce tubulogenesis in human endothelial cells (HUVECs).

HUVECs were grown in conditioned medium derived from MDA-MB-231 cells transfected with ADAMTS-1 siRNA, which created a condition where endothelial cells could be cultured in media with reduced levels of ADAMTS-1. In this situation, tubular network formation was increased as compared to HUVECs grown with MDA-MB-231 control cells (Figure 6B). We next measured the length of the tubes formed in the tubulogenesis 

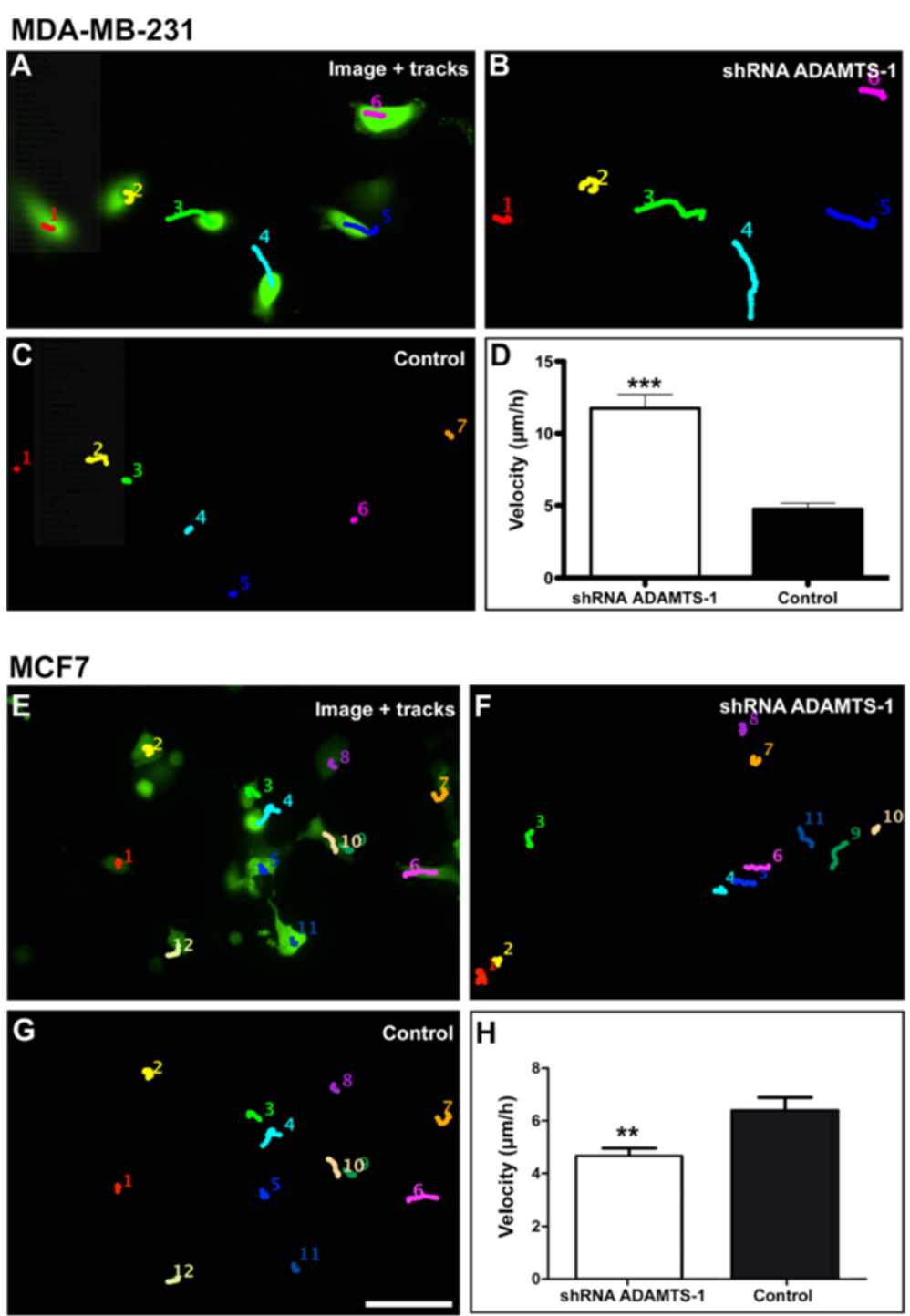

Figure 5 ADAMTS-1 knockdown increased the velocity of MDA-MB-231 cells, as shown by time-lapse video microscopy. The cells were transfected with either ADAMTS-1 shRNA-GFP (treated) or non-silencing shRNA-GFP (controls). The fluorescent images were superimposed with the MDA-MB-231 cell trajectories (A, color tracks) and are illustrated as manual markings on the same cell at different time points. The cells were recorded after 4.5 hours. The treated cells (B) demonstrated longer trajectories over time as compared to the controls (C). The velocity of the treated cells was also higher as compared to the controls (D). The asterisks indicate significant data in comparison to the controls $(p<0.001)$. The results in B represent the mean \pm standard error obtained from 6 experiments. Scale bar: $100 \mu \mathrm{m}$. ADAMTS-1 knockdown decreased the velocity of MCF7 cells, as shown by time-lapse video microscopy. The cells were transfected with either ADAMTS-1 shRNA-GFP (treated) or non-silencing shRNA-GFP (controls). The fluorescent images were then superimposed with MCF7 cell trajectories (color tracks) and are illustrated as manual markings on the same cell at different time points $(\mathbf{E})$. The cells were recorded after 4.5 hours. The treated cells $(\mathbf{F})$ presented shorter trajectories over time as compared to the controls $(\mathbf{G})$, and the velocity of the treated cells was lower in comparison to the controls $(\mathbf{H})$. The asterisks indicate significant data in comparison to the controls $(p<0.01)$. The results in D represent the mean \pm standard error obtained from 6 experiments. Scale bar: $100 \mu \mathrm{m}$.

assays and determined that tube formation with conditioned medium from MDA-MB-231 cells and reduced ADAMTS-1 expression was increased by 4 -fold as compared to the controls (Figure 6C).

Recent reports have also shown that VEGF is related to the migration of cancer cells [13-18]. Therefore, we hypothesized that the differences between MCF7 cell migration and MDA-MB-231 cell migration may be associated with VEGF expression levels in these cell lines. Thus, we performed immunoblots to compare VEGFR2 protein expression levels between MCF7 and MDA-MB231 cells, and we found that VEGFR2 expression levels in MDA-MB-231 cells were 1.5-fold higher than in MCF7 cells (Figure 6D). 

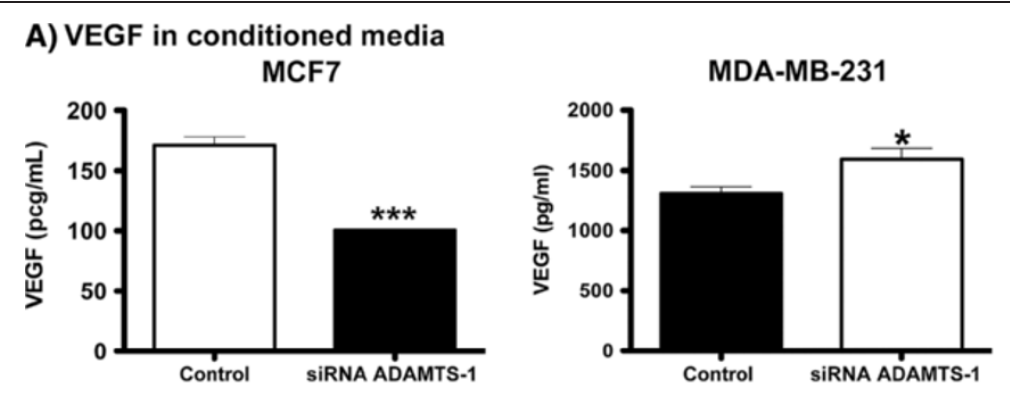

\section{B) Tubulogenesis}
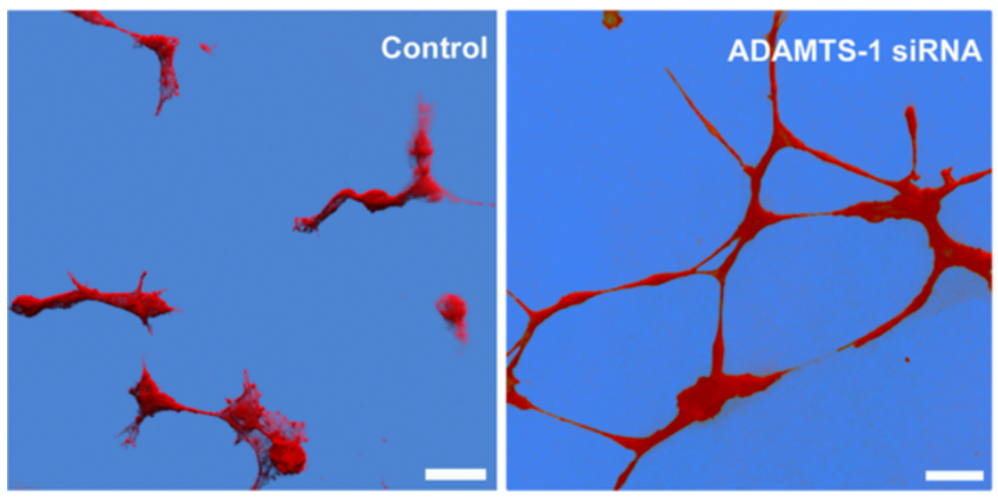

\section{C) Tube length}

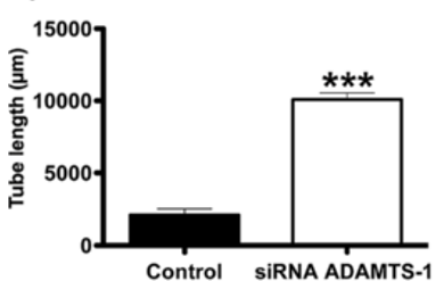

D) VEGFR2 expression

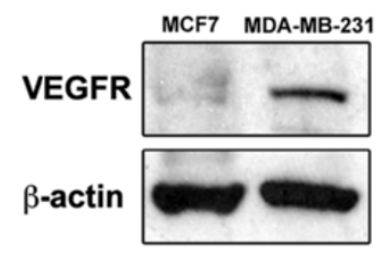

Figure 6 Decreased ADAMTS-1 induced increased VEGF levels in conditioned medium from MDA-MB-231 cells (A) and tubulogenesis in HUVECs (B). ELISAs were carried out to quantify the VEGF levels in conditioned medium from MDA-MB-231 or MCF7 cells treated with siRNA targeting ADAMTS-1. MDA-MB-231 cells with reduced ADAMTS-1 expression exhibited a statistically significant increase in VEGF in conditioned medium (A, right graph). Conversely, MCF7 cells with ADAMTS-1 silenced by siRNA showed a decrease in VEGF levels in conditioned medium (A, left graph). HUVECS were grown in conditioned medium derived from MDA-MB-231 cells transfected with ADAMTS-1 siRNA. In this situation, tubulogenesis was induced after 6 hours (B, right panel). The control cells (medium from cells transfected with control siRNA) showed no particular architecture (B, left panel), whereas tube length was increased in HUVECs treated with conditioned medium derived from MDA-MB-231 cells transfected with ADAMTS-1 siRNA (C). The immunoblot shows the difference in VEGFR expression between MDA-MB-231 and MCF7 cells (D). The asterisks indicate significant data in comparison to the control $\left({ }^{*} p<0.05 ;{ }^{* *} p<0.001\right)$. The panels in (B) represent volumetric renderings from at least 10 confocal optical sections (software Imaris 7.1). The results in $(\mathbf{C})$ represent the mean \pm standard error of three experiments carried out at least three times. Scale bars: $50 \mu \mathrm{m}$.

\section{VEGF and ADAMTS- 1 in cell migration and invasion}

To analyze the putative role of VEGF during the cell migration of ADAMTS-1 siRNA-treated cells, we carried out cell migration and invasion assays. In these assays, a VEGF blocking antibody was included. We found that decreasing ADAMTS-1 levels enhanced both the migration and invasion of MDA-MB-231 cells. Furthermore, treatment with a VEGF blocking antibody partially rescued both cell migration and invasion to the levels obtained with the control (Figure 7A-B).

Furthermore, ADAMTS-1 knockdown increased invadopodia activity in comparison to control siRNA-treated cells, as shown using a fluorescent gelatin assay (Figure 8A-F). The digested gelatin areas (black spots in the fluorescent background) were measured using Image J, and MDA-MB231 cells treated with ADAMTS-1 siRNAs exhibited at least a 5-fold increase in digested areas as compared to the controls.

\section{Discussion}

ADAMTS-1 expression decreased in human breast tumors in vivo, mainly in triple negative cases (ER-, PR-, and Her-2), and ADAMTS-1 knockdown was shown to stimulate migration, invasion and invadopodia formation 


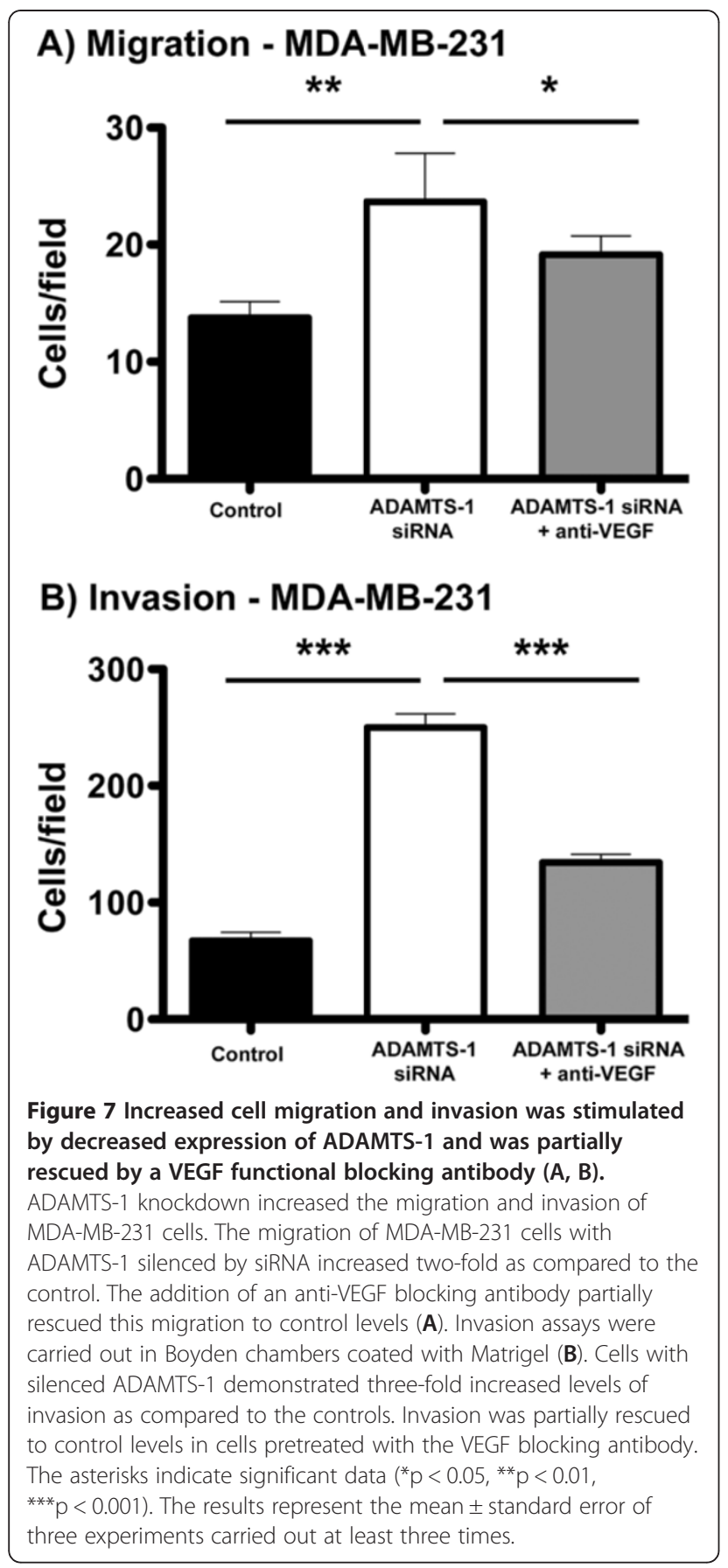

in breast cancer cells in vitro. Our series of experiments further suggest that VEGF is involved in these effects of ADAMTS-1 in breast cancer cells. To our knowledge, this is the first report establishing this relationship in human breast cancer cells.

ADAMTS-1 (a disintegrin and metalloproteinase with thrombospondin motifs) was the first described isoform in this family [19], and this identification was based on its elevated expression in cachexia-inducing adenocarcinomas in mice. Its multidomain structure links this secreted protein to various cellular functions; for example, it is a potent inhibitor of angiogenesis and plays an important role in follicular rupture and ovulation [20] and urogenital development, as demonstrated by the characteristics of knockout animal models [21].

Altered ADAMTS-1 expression has been reported in different types of tumors, including breast cancer $[11,12]$. However, the role of ADAMTS-1 in human breast cancer is not fully understood and requires further investigation. Lu et al. (2009) [22] reported that ADAMTS-1 was overexpressed in $39.7 \%$ of breast tumors, and these authors further demonstrated that ADAMTS-1 overexpression was associated with an increased risk of bone metastasis. Similarly, we observed variable levels of ADAMTS-1 mRNA expression in a series of primary breast tumors. However, the immunolocalization of ADAMTS-1 showed that the expression of this molecule was lower in triple negative tumors as compared to normal tissues. In this study, we compared the distribution of ADAMTS-1 in tumors of different clinical stages, which may explain the apparent discrepancies between our findings and previous data. The previous study by Lu et al. analyzed an array of breast cancer tissues without any consideration for the stage of the tumor. Therefore, we considered tumor stage in our analysis and also evaluated the expression of ADAMTS-1 in the tumor stroma, which was reduced in higher-staged tumors.

Immunoblots comparing normal tissue and cancer tissue revealed that ADAMTS-1 could be detected in four different bands: the $110 \mathrm{kDa}$ band, which likely represents total protein; the $80 \mathrm{kDa}$, which may characterize the protease without the pro-domain or the activated form; and two smaller bands, which may correspond to activated ADAMTS-1 with additional proteolytic processing [23,24]. We also observed that the $80 \mathrm{kDa}$ ADAMTS-1 band was decreased in breast tumors as compared to adjacent normal tissue samples. Thus, a reduction in ADAMTS-1, as determined by immunohistochemistry, may represent a decrease in the $80 \mathrm{kDa}$ ADAMTS-1 band. With regard to the in vitro expression of ADAMTS-1, both the MCF7 and MDA-MB-231 cell lines exhibited a prominent ADAMTS-1 $80 \mathrm{kDa}$ band in conditioned medium.

Our results showed that MDA-MB-231 cells with reduced ADAMTS-1 expression demonstrated increased migration, velocity and invasion. In cancer progression, cell-to-cell detachment from the primary tumor and the acquisition of a motile phenotype are required for cells to become invasive and colonize distant organs, thereby producing a metastasis [25]. The spread of cancer cells to distant sites in the body is the major cause of death for cancer patients [26,27], and one major challenge in cancer therapy is to inhibit the spread of tumor cells from the primary tumor site to distant organs [28]. 

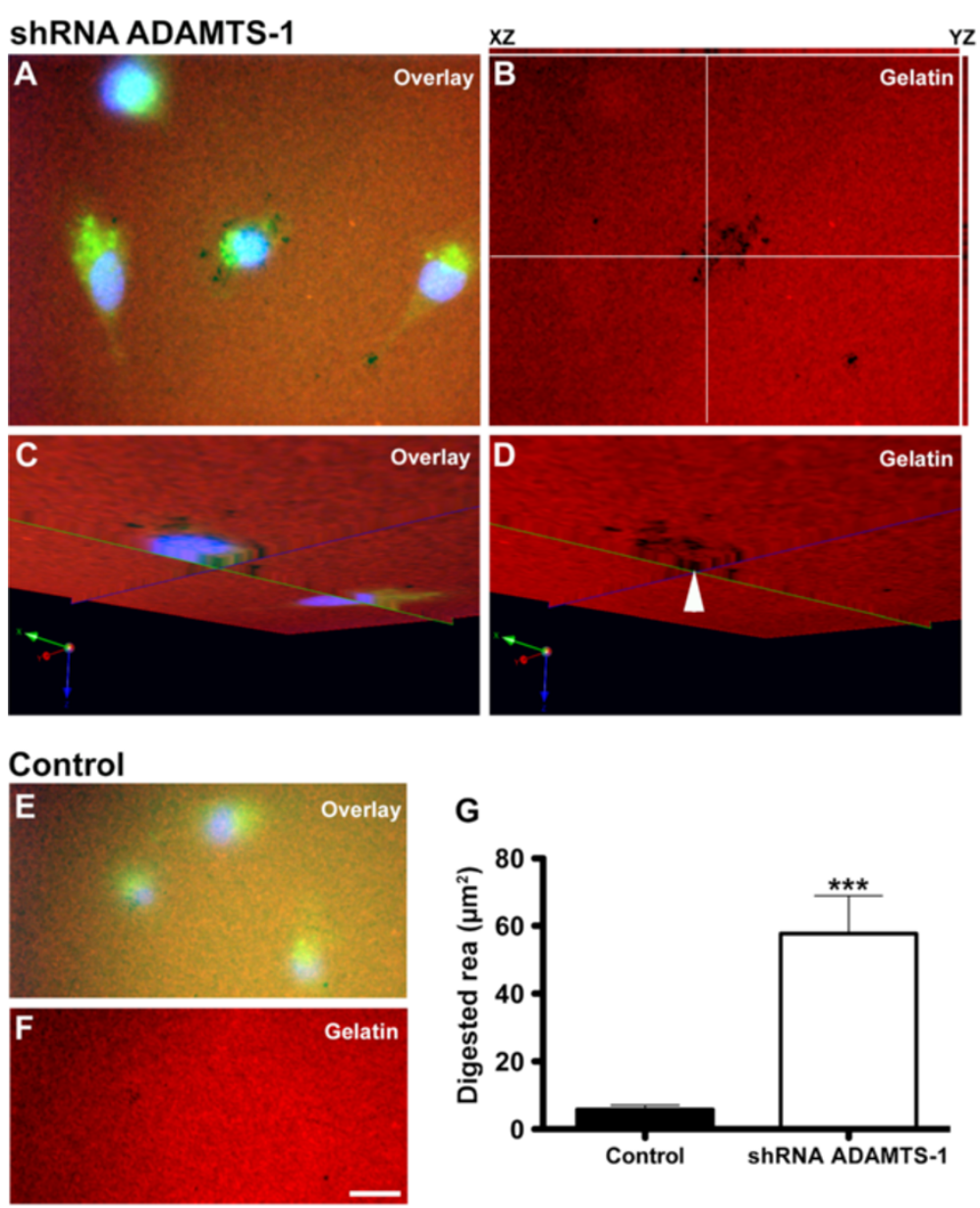

Figure 8 ADAMTS-1 knockdown increased invadopodia formation in MDA-MB-231 cells. Cells were grown on gelatin-Alexa-567 for 6 hours. Image $\mathbf{A}$ is a merged image of the blue (nuclei stained with DAPI), red (gelatin-Alexa 567) and green (ADAMTS-1 shRNA-GFP) channels. Z-projections of 10 optical sections together with orthogonal projections onto XZ (top) and YZ (left) planes were generated (B). The white lines in (B) indicate points of the $X Y$ image projected to generate orthogonal (perpendicular) $X Z$ (top) and $Y Z$ (right) planes. Orthogonal projections of $X Z$ and $Y Z$ illustrate digestion at the ventral surfaces of cells. Substrate digestion could be clearly observed when planes XY, XZ and YZ were viewed in the same volume ( $\mathbf{C}$ and $\mathbf{D}$, arrowhead). Images $C$ and $D$ were rotated to observe the cell's ventral surface. The control samples (treated with non-silencing shRNA-GFP) show discrete matrix digestion (E and $\mathbf{F}$ ). The area measurements demonstrate that ADAMTS-1 knockdown significantly increased matrix digestion $(\mathbf{G})$. Digested areas in close proximity to the cell ventral surfaces indicate that ADAMTS-1 knockdown induced invadopodia activity. The asterisks indicate significant data in comparison to the control $\left({ }^{*} p<0.05,{ }^{* * *} p<0.001\right)$. A total of 10 random fields were imaged per experimental group. Scale bar: $10 \mu \mathrm{m}$.

Previous reports have acknowledged the role of ADAMTS-1 in cell migration. Krampert et al. (2005) [29] studied the role of ADAMTS-1 in the healing of skin wounds. In this model, they observed that ADAMTS-1 played different roles in fibroblast migration depending on the concentration; a decrease in the level of this protein stimulated cell migration via the proteolytic activity of ADAMTS-1.

The effects of ADAMTS-1 knockdown on cell migration and invasion seem to be related to VEGF, as MDA-MB-231 cells with reduced ADAMTS-1 expression showed increased levels of VEGF in conditioned medium. The relationship between VEGF and ADAMTS-1 was recently reported, and the carboxyl-terminal domain of ADAMTS-1 was shown to be responsible for binding and sequestering VEGF [7]. This sequestration of VEGF by ADAMTS likely inhibits various functions of VEGF, such as its role in cell migration and invasion.

It has been described that ADAMTS-1 sequesters VEGF [7]. VEGF is known to enhance migration and invasion $[13,15,16,18]$. We then carried out combined multi-tiered experiments to relate the role of ADAMTS and VEGF during cell migration and invasion. ADAMTS1 knockdown in MDA-MB-231 cells resulted in a decrease in ADAMTS-1 protease activity in conditioned medium. Furthermore, cells with reduced ADAMTS-1 expression demonstrated increased levels of VEGF in conditioned medium. Taken together, these results suggest that 
ADAMTS-1 knockdown decreased the presence of this protease in the conditioned medium of MDA-MB-231 cells, thus preventing the sequestration of VEGF and rendering this growth factor available to exert its cellular effects, including migration and angiogenesis $[13,15,16,18]$.

To analyze the putative role of VEGF in the cell migration of MDA-MB-231 cells, we carried out migration and invasion assays in MDA-MB-231 cells with reduced ADAMTS-1 expression. To assess the effect of VEGF, we used a VEGF blocking antibody and found that ADAMTS-1 knockdown increased migration and invasion, as expected. However, treatment with blocking antibodies partially rescued both cell migration and invasion, and these results suggest a close relationship between ADAMTS-1 and VEGF in regulating cell migration and invasion.

The evidence presented here establishes a relationship between ADAMTS-1 and VEGF, and our results also indicated that VEGF in conditioned medium from MDA-MB231 cells with ADAMTS-1 silenced initiated tubulogenesis in HUVEC cells. ADAMTS-1 has been described as a protease with angioinhibitory properties [6] that significantly blocks VEGFR2 phosphorylation and suppresses endothelial cell proliferation. In addition, the inhibition of ADAMTS-1-related angiogenesis is related to the sequestering of VEGF.

VEGF also induces invadopodia formation by increasing the activity of MMP-2, MMP-9 and MT1-MMP [15]. MDA-MB-231 cells with ADAMTS-1 knockdown demonstrated increased invasion in Boyden chambers, and cells with reduced ADAMTS-1 expression also demonstrated increased invadopodia formation. Therefore, MDAMB-231 cells with depleted levels of ADAMTS-1 may increase the availability of VEGF, which could enhance invadopodia formation and/or activity.

Various authors have demonstrated invadopodia formation and/or activity in MDA-MB-231 cells using a variety of approaches. For example, invadopodia activity in MDA-MB-231 cells has been reported in srctransformed cells [30,31], in cells cultured on fibronectin [32] and in cells treated with growth factors [33]. Most of these studies were carried out in cells grown for at least 16 hours; in contrast, our results revealed invadopodia activity over a short timeframe as well as increased matrix digestion in MDA-MB-231 cells when ADAMTS1 is knocked down.

Cancer cells rely on invadopodia to initiate invasive activity [30,34], as these formations are enriched with actin filaments (F-actin) and components needed for actin assembly, including neural-Wiskott Aldrich Syndrome protein (N-WASP) and cortactin [30,35-37]. Therefore, it was not surprising that the functional knockdown of ADAMTS-1 stimulated the migratory and invasive activity of MDA-MB-231 cells. On the other hand, ADAMTS-1 knockdown had the opposite effect on MCF7 cells and reduced their migratory activity. This discrepancy could be the result of different biological behaviors between these cell lines. It is well known that MCF7 cells express estrogen and progesterone receptors, while MDA-MB-231 cells do not. Thus, MDA-MB-231 cells may be more aggressive and invasive as compared to MCF7 cells.

Another possible explanation for these differences in migration and invasion could be related to VEGF and VEGF receptor levels. VEGFR2 expression levels in MDA-MB-231 cells were 1.5-fold higher in comparison to MCF7 cells, whereas MDA-MB-231 cells with reduced ADAMTS-1 expression showed augmented VEGF levels in the conditioned medium as compared to the controls. Taken together, these results suggest that MDA-MB231 cells possess suitable machinery to interact with VEGF, a growth factor important for migration and invasion $[13,15-18]$.

\section{Conclusions}

Our study was the first to describe lower levels of ADAMTS-1 protein in triple-negative breast cancer cases (ER-, PR-, and Her-2), and our immunoblot analyses indicated that ADAMTS-1 expression was reduced in tumors in comparison to contralateral normal tissues. In addition, ADAMTS-1 knockdown stimulated migration, invasion and invadopodia formation in breast cancer cells in vitro. This series of experiments suggested that VEGF is involved in ADAMTS-1 effects on breast cancer cells; the diagram depicted in Figure 9 illustrates our current understanding of ADAMTS-1 in breast cancer. In normal cells, the extracellular environment is enriched with ADAMTS-1, and this protease sequesters VEGF, thus preventing the effects of this growth factor on migration and invasion. On the other hand, breast cancer cells exhibit lower levels of ADAMTS-1 in the extracellular matrix, and in this scenario, VEGF is no longer sequestered and becomes freely available to bind VEGFR, which increases breast cancer cell migration and invasion. Future investigations are needed to determine the precise mechanisms by which ADAMTS-1 and VEGF regulate the invasiveness of cancer cells.

\section{Material and methods}

\section{Tissue samples, patient characteristics, RNA extraction} and quantitative real-time PCR (qPCR)

Sixty tumor samples and 20 normal tissues adjacent to the tumors were obtained from 60 breast cancer patients at the Hospital do Câncer, A. C. Camargo, São Paulo, Brazil. Patient age ranged from 23 to 85 years (median 54 years). Tumor samples were dissected to remove any residual normal tissue before freezing and storing the samples in liquid nitrogen. The largest diameter of the tumors was recorded. Microscopic examinations were 


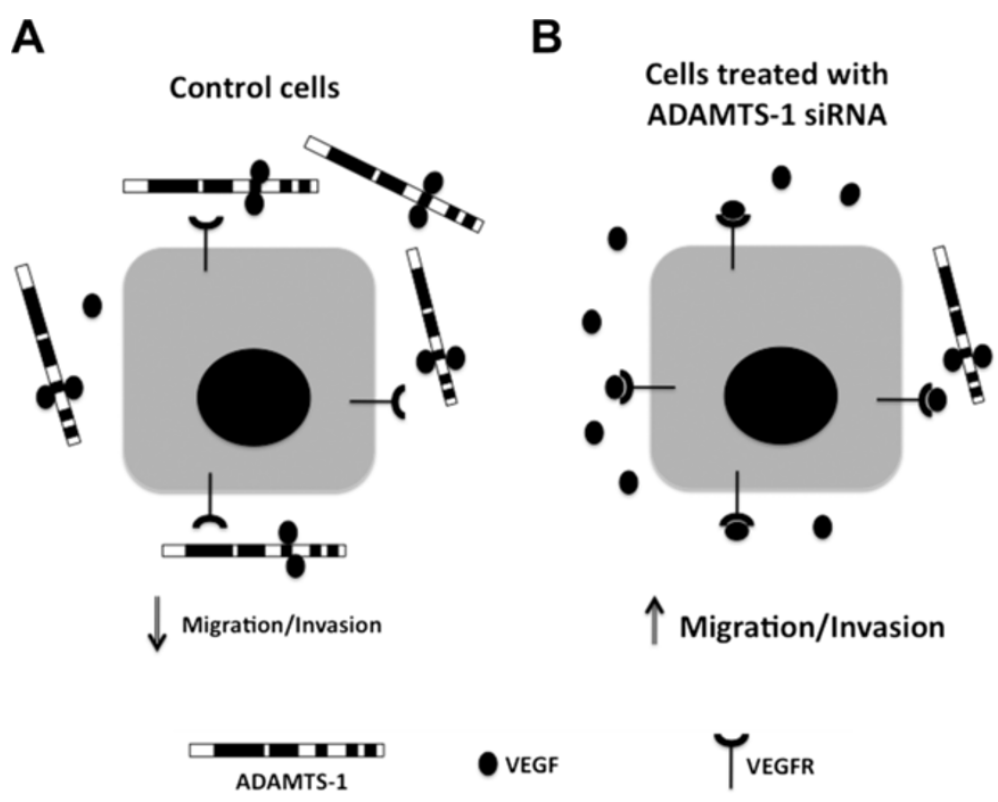

Figure 9 Schematic diagram summarizing our current understanding of the role of ADAMTS-1 in breast cancer cells. In normal cells (A), the extracellular environment is enriched for ADAMTS-1. This protease sequesters VEGF, thus preventing the effects of this growth factor on migration and invasion. In contrast, tumor cells (B) exhibit lower levels of ADAMTS-1 in the extracellular matrix. In this scenario, VEGF is no longer sequestered and becomes freely available to bind VEGFR, thereby increasing the migration and invasion of breast cancer cells.

performed to determine the average number of lymph node metastases in 24 patients. Tumor metastasis into the lymph nodes was detected in 40 patients. Histopathological review of all the tumor slides was performed to confirm the diagnosis. All tumors were classified according to the WHO Histological Typing of Breast Tumors. Infiltrating ductal carcinomas were studied, and the clinical stage was assigned based on the UICC TNM (tumor, nodes, metastases) staging system.

The Institutional Ethics Committee approved this study, and all subjects provided informed consent.

Tissue specimens were pulverized under liquid nitrogen using a Frozen Tissue Pulverizer (Thermovac Industries Corporation, Copiague, NY, USA), and total RNA was extracted using the acid guanidinium thiocyanatephenol-chloroform method [38]. Ten micrograms of total RNA, previously treated with DNaseI, was reverse transcribed using a High Capacity cDNA Archive Kit (Applied Biosystems, Carlsbad, CA, USA). qPCR was performed using an Applied Biosystems 7500 Real-Time PCR System, and each cDNA sample was analyzed in duplicate. The PCR reactions were carried out in a total volume of $25 \mu \mathrm{l}$ according to the manufacturer's instructions for the SYBR Green PCR Core reagent (PE Applied Biosystems). The following PCR primers were used: ADAMTS-1 forward, 5'- TGTGGTGTTTGCGGGGGA AATG-3' and reverse, 5'- TCGATGTTGGTGGCTCCA GTT -3'; and glyceraldehyde-3-phosphate dehydrogenase
$(G A P D H)$ forward, 5'-CCTCCAAAATCAAGTGGGGC G-3' and reverse, 5'-GGGGCAGAGATGATGACCCTT3'. The algorithm geNorm was used to define the calibrator gene among the RLP13, HPRT, ACTB and GAPDH transcripts. As a result, the relative gene expression was normalized, with GAPDH expression serving as the internal control. The average value from two groups of 10 normal tissue samples served as a reference sample. The results were expressed as the $n$-fold difference in target gene expression relative to the expression of the GAPDH gene and the reference sample. The relative expression was calculated using the $2^{-\Delta \Delta C T}$ method $(C T=$ fluorescence threshold value; $\triangle \mathrm{CT}=\mathrm{CT}$ of the target gene - $\mathrm{CT}$ of the reference gene (GADPH); $\Delta \Delta C T=\Delta C T$ of the tumor sample - $\Delta \mathrm{CT}$ of the reference sample).

\section{Immunohistochemical analysis}

Tissue microarray slides from normal human and breast cancer samples were obtained from Imgenex (San Diego, $\mathrm{CA}$; IMH-364). Fifty-nine samples in 4- $\mu \mathrm{m}$ sections were analyzed, including 35 cases of invasive ductal carcinoma (IDC), 1 case of sarcomatoid carcinoma, 1 case of intraductal papillary carcinoma, 1 case of atypical medullary carcinoma, 1 case of metaplastic carcinoma, 1 case of ductal carcinoma in situ, 10 cases of cancer metastasis and 9 samples of normal breast tissue adjacent to cancer tissue. Among these samples, 19 were triple-negative tumors, with 7 ER- and PR-positive cases. For antigen 
retrieval, $10 \mathrm{mM}$ of citrate buffer ( $\mathrm{pH}$ 6.0) with $0.05 \%$ Tween 20 was applied for 30 minutes at $100^{\circ} \mathrm{C}$. Then, the EnVision method (EnVision; Dako Corp., Carpinteria, CA, USA) was used to analyze the TMAs, and diaminobenzidine served as the chromogen.

ImageJ public domain software (http://rsb.info.nih.gov/ij/) was used for image analysis. The DAB channel was separated by the color deconvolution plugin. The stained areas were segmented and measured. Quantification involved either DAB labeling divided by hematoxylin staining or scoring the labeled intensity of tumor cells or stroma. Immunohistochemical staining was quantitatively assessed by three independent observers (VMF, JBA, RGJ) with minimal interobserver variability $(<5 \%)$.

\section{Cell lines and transfection}

MCF7 and MDA-MB-231 cells were cultured in Dulbecco's Modified Eagle's Medium-F12 (DMEM-F12, Sigma) supplemented with 10\% fetal bovine serum (FBS; Cultilab, Campinas, SP, Brazil). Human umbilical vascular endothelial cells (HUVECs) were cultured in 199 Medium supplemented with LSGS (Gibco). The cells were maintained in $25 \mathrm{~cm}^{2}$ flasks in a humidified atmosphere of $5 \% \mathrm{CO}_{2}$ at $37^{\circ} \mathrm{C}$.

MCF7 and MDA-MB-231 cells were transfected with commercially available siRNA targeting ADAMTS-1 (Santa Cruz Biotechnology Inc., Santa Cruz, CA, USA), according to the manufacturer's instructions. One day prior to transfection, subconfluent MCF7 and MDA-MB231 cells were cultured in DMEM supplemented with $10 \%$ FBS without antibiotic-antimycotic solution. The cells were incubated with a complex formed by the siRNA (50 nM), transfection reagent (Lipofectamine 2000, Invitrogen) and transfection medium (Opti-MEM I, Invitrogen) for $30 \mathrm{~h}$ at $37^{\circ} \mathrm{C}$.

SureSilencing shRNA plasmids for human ADAMTS-1 with GFP (\#KH01149G, SABiosciences, Frederick, MD, USA) were used for the time-lapse experiments.

Cells transfected with scrambled siRNA or shRNA served as controls. These assays were performed in triplicate.

\section{Time-lapse fluorescence microscopy}

For the time-lapse fluorescence microscopy, shRNAGFP was used to silence ADAMTS-1, as previously described. Cells transfected with scrambled interference RNA served as control. The migration was determined using a time-lapse fluorescence microscope with an Olympus IX 81 inverted microscope equipped with an Orca R2 CCD camera. Video recordings were conducted using Cell Observer image software (Olympus). Treated and control cells were maintained at $37^{\circ} \mathrm{C}$ in a temperature-controlled chamber, and images were collected every 5 minutes (over a total of 4 hours and 30 minutes). MTrack J plugin (Image J software) was used to measure cell velocity.

\section{Migration and invasion assays}

Transwell inserts (with $8 \mu \mathrm{m}$ pores) in 12-well plates (BD Biosciences) were used for the migration and invasion assays, where ADAMTS-1 was silenced by siRNA (Santa Cruz).

In the migration assays, treated and control cells $\left(10^{5}\right)$ were plated into the upper chamber containing $1 \mathrm{~mL}$ of DMEM-F12 without serum. The lower chamber was filled with $1.5 \mathrm{~mL}$ of DMEM-F12. After 24 hours in culture, the cells were fixed with $4 \%$ paraformaldehyde and post-fixed with $0.2 \%$ crystal violet in $20 \%$ methanol. Cells on the upper side of the filter were removed with a cotton swab. The migrating cells on the lower side of the filter were photographed and counted. These experiments were performed in triplicate and were repeated at least three times.

For the invasion assays, the filters were coated with $10 \mu \mathrm{l}$ of Matrigel $(10-13 \mathrm{mg} / \mathrm{ml})$. Treated and control cells $\left(10^{5}\right)$ were plated into the upper chamber containing $1 \mathrm{~mL}$ of DMEM-F12 without serum. The lower chamber was filled with $1.5 \mathrm{~mL}$ of DMEM-F12. After 48 hours in culture, the cells were fixed and stained. The cells on the upper side of filter were removed, as described above, whereas the invading cells on the lower side of the filter were photographed and counted. These experiments were performed in triplicate and were repeated at least three times.

To determine the role of VEGF in migration and invasion following ADAMTS-1 depletion, cells with silenced ADAMTS-1 $\left(10^{5}\right)$ and controls were plated into the upper chamber containing $1 \mathrm{~mL}$ of DMEM-F12 without serum. The cells were incubated with anti-VEGF blocking antibody (1 $\mu \mathrm{g} / \mathrm{ml} \mathrm{R \& D}$ Systems) or non-specific mouse IgG $(1 \mu \mathrm{g} / \mathrm{ml}$ Millipore). The lower chamber was filled with $1.5 \mathrm{~mL}$ of DMEM-F12 supplemented with $10 \%$ of serum. The migration and invasion assays were conducted as previously described.

\section{ELISA}

The level of VEGF in conditioned medium was quantified using MCF7 and MDA-MB-231 cells and the Human VEGF ELISA kit (Invitrogen). Cells were transfected with either ADAMTS-1 siRNA or control siRNA, followed by serum starvation for $24 \mathrm{~h}$. The conditioned media were collected and centrifuged at 1,000 rpm for $5 \mathrm{~min}$ at $4^{\circ} \mathrm{C}$ to remove cellular debris. The conditioned medium was then analyzed by ELISA according to the manufacturer's instructions, and the results were expressed in $\mathrm{pg} / \mathrm{mL}$.

\section{Tubulogenesis assay}

ADAMTS-1 expression was silenced by siRNA in MDAMB-231 cells, and cells that were transfected with scrambled siRNAs served as controls. We obtained conditioned 
medium from treated and control cells, as previously described, and this conditioned medium was used to induce tubulogenesis in human endothelial cells (HUVECs).

Three-dimensional HUVEC cultures were seeded on a solidified layer of reduced growth factor (RGF) Matrigel, approximately 1-2 $\mathrm{mm}$ in thickness. A round 13-mm coverslip coated with $20 \mu \mathrm{l}$ of RGF-Matrigel (Trevigen, Gaithersburg, MD, kindly provided by Dr. Matthew Hoffman, NIDCR, NIH) was placed in each well of a 24-well plate. Then, a 100- $\mu$ l drop of the cell suspension $\left(3.5 \times 10^{5}\right.$ cells $\left./ \mathrm{ml}\right)$ was placed on top of the Matrigel and incubated in HUVEC complete medium. After two hours, the non-adherent cells were washed, and the complete medium was replaced with serum-free medium and conditioned for 24 hours with confluent two-dimensional cultures of either control or siRNA-treated MDAMB-231 cells. The HUVEC cells remained in these conditions for 4 hours, followed by fixation in $4 \%$ paraformaldehyde in PBS, permeabilization with $0.5 \%$ Triton $\mathrm{X}-100$, actin staining with rhodamine-phalloidin (Invitrogen-Molecular Probes, Eugene, OR) and mounting with ProLong-DAPI (Invitrogen). The images were acquired using a Zeiss LSM 510 laser scanning confocal microscope (Carl Zeiss, Oberkochen, Germany) and analyzed using Imaris 7.1 (Bitplane Inc, South Windsor, CT, USA) and Wimasis (Wimasis GmbH, Munich, Germany).

\section{Fluorescent substrate degradation assay}

To assess the role of ADAMTS- 1 in MDA-MB-231 cell invadopodia formation, we carried out a fluorescent gelatin substrate degradation assay. The substrate was prepared using gelatin conjugated to Alexa 568 (Invitrogen, Eugene, OR, USA), and the conjugation followed the manufacturer's instructions. MDA-MB-231 cells $(2 \times$ $10^{4} / \mathrm{mL}$ ) that were transfected with ADAMTS-1 shRNA were plated onto the fluorescent gelatin substrate and incubated in DMEM with $10 \% \mathrm{FBS}$ at $37^{\circ} \mathrm{C}$ for 6 hours. The control cells were treated with scrambled nonsilencing shRNA. Treated and control cells were fixed in $4 \%$ paraformaldehyde in PBS and mounted using ProLong containing DAPI (Invitrogen).

The images were obtained with an Axiophot widefield fluorescence microscope using a 63x PlanApo 1.4 NA objective (Carl Zeiss) and acquired using a digital CCD monochromatic camera (CoolSnap HQ2, Photometrics Inc, Tucson, AZ, USA). To assess matrix digestion spots in the fluorescent substrate, at least ten $\mathrm{Z}$ sections per sample field were obtained using a piezoelectric device (PIFOC, Physik Instrumente, Germany) coupled to the objective. The microscope and devices were controlled using the Metamorph Premier 7.6 software (Molecular Devices, Sunnyvale, CA, USA).

We also examined shRNA-GFP-transfected cells (green channel) while screening for superimposed digested areas (dark spots) in the fluorescent gelatin matrix (red channel). ImageJ was used to measure the degraded areas. The red channel (fluorescent gelatin) was separately processed using a threshold tool to calculate the digested area $\left(\mu \mathrm{m}^{2}\right)$ per cell. Volocity software (PerkinElmer, Waltham, MA, USA) was used to determine the orthogonal projections and image restorations using deconvolution algorithms.

\section{Western blot}

Western blots were carried out to compare ADAMTS-1 levels in MCF7 and MDA-MB-231 cell lysates and conditioned medium and to verify siRNA transfection efficiency. The cells were lysed in RIPA buffer $(150 \mathrm{mM}$ $\mathrm{NaCl}, 1.0 \% \mathrm{NP}-40,0.5 \%$ deoxycholate, $0.1 \% \mathrm{SDS}, 50 \mathrm{mM}$ Tris $\mathrm{pH}$ 8.0) containing a protease inhibitor cocktail (Sigma). After centrifugation $(10,000 \mathrm{~g})$ for $10 \mathrm{~min}$ at $4^{\circ} \mathrm{C}$, the supernatants were recovered and quantified (BCA kit, Pierce). The samples were resuspended in Laemmli buffer containing $62.5 \mathrm{mM}$ Tris- $\mathrm{HCl} \mathrm{pH} 6.8$, $2 \%$ sodium dodecyl sulphate (SDS), 10\% glycerol, 5\% mercaptoethanol and $0.001 \%$ bromophenol blue. The conditioned medium $(1 \mathrm{~mL})$ was then ethanol-precipitated, and equal amounts $(30 \mu \mathrm{g})$ of the cell lysates were electrophoresed on $10 \%$ polyacrylamide gels. The proteins were transferred to a Hybond ECL nitrocellulose membrane (Amersham) and blocked in TBS with 5\% non-fat milk overnight at $4^{\circ} \mathrm{C}$. Following one wash in TBS with $0.05 \%$ Tween 20 (TBST), the membranes were probed with antibodies against ADAMTS-1 (1:1,000, Abcam 28284), VEGFR2 (1:500, Santa Cruz) and $\beta$-actin (1:2,000, Sigma). The ECL protocol was used to detect proteins on the membrane.

We also used OncoPair INSTA-Blot Breast Tissue membranes (IMB-130a, b, c and e; IMGENEX, San Diego, CA, USA). These ready-to-use PVDF membranes contain denatured protein lysates from ductal carcinomas and were matched with adjacent tissues obtained from seven donors. Each membrane was probed with ADAMTS-1 (Abcam 28284) and alpha-tubulin (Abcam 4074) and revealed using the ECL protocol.

\section{Statistical methods}

The median values of ADAMTS-1 mRNA expression were in accordance with the clinical and pathological variables and were compared using the Kruskal-Wallis and Mann-Whitney tests. For survival analyses, ADAMTS-1 expression was classified as low $(<0.7)$ or high $(\geq 0.7)$ according to its median expression value. The overall survival and disease-free survival rates were calculated using the Kaplan-Meier method, and the curves were compared using the log-rank test. The overall survival and diseasefree survival were calculated from the day of diagnosis to the date of death and to the date in which recurrence was detected, respectively. The significance level was set at $5 \%$ 
for all tests. The statistical analyses were performed using SPSS software 15.0 (SPSS Inc., Chicago, IL). The protein data analyses and statistical significance were obtained using the Wilcoxon matched test. For in vitro experiments, the data were analyzed using Graph Pad Prism 5 software (Graph Pad Software, Inc., San Diego, CA, USA). A Student's $t$ test or ANOVA was used to assess the differences.

\section{Competing interests}

The authors declare that they have no competing interests.

\section{Authors' contributions}

VMF, MAN and GMMS designed this study. FRM and MAN carried out the qPCR analysis of ADAMTS-1 expression in normal and neoplastic tissues. VMF and TAS performed experiments, including immunoblotting in ready-to-use PVDF membranes and analyzed ADAMTS-1 in cell line lysates and conditioned medium (Western blot). VMF, JBA, JJVP and RGJ carried out immunohistochemistry on TMA and performed the data analysis. VMF and JBA performed the experiments with shRNA and the time-lapse migration assay. VMF performed siRNA transfection, migration and invasion assays in Boyden chambers. VMF, RGJ and ESS carried out the tubulogenesis and invadopodia assays. JBA and GMMS performed the confocal microscopy analysis of HUVECs. VMF, MAN and RGJ prepared the manuscript, performed statistical analyses of the data and contributed to discussions and interpretations of the results. All authors have read and approved the final manuscript.

\section{Acknowledgements}

This investigation was supported by The State of São Paulo Research Foundation (FAPESP grants 2006/54963-0, 2006/01026-0, 2008/57103-8, 2010/ 07699-1), and Brazilian National Council for Scientific and Technological Development (CNPq grant 470779/2007-1). The authors also want to thank Dr. Stanley Zucker (Stony Brook University; Stony Brook, NY, USA) and Dr. Rama Khokha (University of Western Ontario, Toronto, Canada) for suggestions made on data analysis.

\section{Author details}

${ }^{1}$ Departamento de Biologia Celular e do Desenvolvimento, Instituto de Ciências Biomédicas, Universidade de São Paulo, Av. Prof. Lineu Prestes 1524, Ed Biomédicas 1 sala 428, São Paulo, SP 05508-000, Brazil. 'Departamento de Análises Clínicas, Faculdade de Ciências Farmacêuticas, Universidade de São Paulo Ribeirão Preto, Av. do Café, s/n, Ribeirão Preto, São Paulo 14040-903, Brazil. ${ }^{3}$ Disciplina de Oncologia, Departamento de Radiologia e Oncologia da Faculdade de Medicina da Universidade de São Paulo, Av. Dr. Arnaldo, 455, 4 andar, São Paulo, SP 01246-903, Brazil. " Faculdade de Odontologia, Instituto de Ciências da Saúde, Programa de Pós-Graduação em Odontologia, Universidade Federal do Pará, Rua Augusto Corrêa, 01, Belém, Pará 66075-110, Brazil.

Received: 21 September 2012 Accepted: 4 January 2013 Published: 5 January 2013

\section{References}

1. Comoglio PM, Trusolino L: Cancer: the matrix is now in control. Nat Med 2005, 11:1156-1159.

2. Kleinman HK, Philp D, Hoffman MP: Role of the extracellular matrix in morphogenesis. Curr Opin Biotechnol 2003, 14:526-532.

3. Rocks N, Paulissen G, El Hour M, Quesada F, Crahay C, Gueders M, Foidart JM, Noel A, Cataldo D: Emerging roles of ADAM and ADAMTS metalloproteinases in cancer. Biochimie 2008, 90:369-379.

4. Pantel K, Brakenhoff RH: Dissecting the metastatic cascade. Nat Rev Cancer 2004, 4:448-456.

5. Brown HM, Dunning KR, Robker RL, Pritchard M, Russell DL: Requirement for ADAMTS-1 in extracellular matrix remodeling during ovarian folliculogenesis and lymphangiogenesis. Dev Biol 2006, 300:699-709.

6. Iruela-Arispe ML, Carpizo D, Luque A: ADAMTS1: a matrix metalloprotease with angioinhibitory properties. Ann N Y Acad Sci 2003, 995:183-190.
7. Luque A, Carpizo DR, Iruela-Arispe ML: ADAMTS1/METH1 inhibits endothelial cell proliferation by direct binding and sequestration of VEGF165. J Biol Chem 2003, 278:23656-23665.

8. $\mathrm{Ng}$ YH, Zhu H, Pallen CJ, Leung PC, MacCalman CD: Differential effects of interleukin-1 beta and transforming growth factor-beta1 on the expression of the inflammation-associated protein, ADAMTS-1, in human decidual stromal cells in vitro. Hum Reprod 2006, 21:1990-1999.

9. Kuno K, Okada Y, Kawashima $\mathrm{H}$, Nakamura $\mathrm{H}$, Miyasaka M, Ohno H, Matsushima K: ADAMTS-1 cleaves a cartilage proteoglycan, aggrecan. FEBS Lett 2000, 478:241-245.

10. Rocks N, Paulissen G, Quesada-Calvo F, Munaut C, Gonzalez ML, Gueders M, Hacha J, Gilles C, Foidart JM, Noel A, Cataldo DD: ADAMTS-1 metalloproteinase promotes tumor development through the induction of a stromal reaction in vivo. Cancer Res 2008, 68:9541-9550.

11. Masui T, Hosotani R, Tsuji S, Miyamoto Y, Yasuda S, Ida J, Nakajima S, Kawaguchi M, Kobayashi H, Koizumi M, et al: Expression of METH-1 and METH-2 in pancreatic cancer. Clin Cancer Res 2001, 7:3437-3443.

12. Porter S, Scott SD, Sassoon EM, Williams MR, Jones JL, Girling AC, Ball RY, Edwards DR: Dysregulated expression of adamalysin-thrombospondin genes in human breast carcinoma. Clin Cancer Res 2004, 10:2429-2440.

13. Chen CH, Lai JM, Chou TY, Chen CY, Su L, Lee YC, Cheng TS, Hong YR, Chou CK, Whang-Peng J, et al: VEGFA upregulates FLJ10540 and modulates migration and invasion of lung cancer via PI3K/AKT pathway. PLoS One 2009, 4:e5052.

14. Green CE, Liu T, Montel V, Hsiao G, Lester RD, Subramaniam S, Gonias SL, Klemke RL: Chemoattractant signaling between tumor cells and macrophages regulates cancer cell migration, metastasis and neovascularization. PLoS One 2009, 4:e6713.

15. Lucas JT Jr, Salimath BP, Slomiany MG, Rosenzweig SA: Regulation of invasive behavior by vascular endothelial growth factor is HEF1dependent. Oncogene 2010, 29:4449-4459.

16. Oommen S, Gupta SK, Vlahakis NE: Vascular endothelial growth factor A (VEGF-A) induces endothelial and cancer cell migration through direct binding to integrin \{alpha\}9\{beta\}1: identification of a specific \{alpha\}9 \{beta\}1 binding site. J Biol Chem 2011, 286:1083-1092.

17. Prager GW, Lackner EM, Krauth MT, Unseld M, Poettler M, Laffer S, Cerny-Reiterer S, Lamm W, Kornek GV, Binder BR, et al: Targeting of VEGFdependent transendothelial migration of cancer cells by bevacizumab. Mol Oncol 2010, 4:150-160.

18. Zhu C, Xiong Z, Chen X, Lu Z, Zhou G, Wang D, Bao J, Hu X: Soluble vascular endothelial growth factor (VEGF) receptor-1 inhibits migration of human monocytic THP-1 cells in response to VEGF. Inflamm Res 2011, 60:769-774.

19. Kuno K, Kanada N, Nakashima E, Fujiki F, Ichimura F, Matsushima K: Molecular cloning of a gene encoding a new type of metalloproteinasedisintegrin family protein with thrombospondin motifs as an inflammation associated gene. J Biol Chem 1997, 272:556-562.

20. Robker RL, Russell DL, Espey LL, Lydon JP, O'Malley BW, Richards JS: Progesterone-regulated genes in the ovulation process: ADAMTS-1 and cathepsin L proteases. Proc Natl Acad Sci U S A 2000, 97:4689-4694

21. Shindo $T$, Kurihara $H$, Kuno $K$, Yokoyama H, Wada $T$, Kurihara $Y$, Imai T, Wang Y, Ogata M, Nishimatsu H, et al: ADAMTS-1: a metalloproteinasedisintegrin essential for normal growth, fertility, and organ morphology and function. J Clin Invest 2000, 105:1345-1352.

22. Lu X, Wang Q, Hu G, Van Poznak C, Fleisher M, Reiss M, Massague J, Kang Y: ADAMTS1 and MMP1 proteolytically engage EGF-like ligands in an osteolytic signaling cascade for bone metastasis. Genes Dev 2009, 23:1882-1894.

23. Liu YJ, Xu Y, Yu Q: Full-length ADAMTS-1 and the ADAMTS-1 fragments display pro- and antimetastatic activity, respectively. Oncogene 2006, 25:2452-2467.

24. Rehn AP, Birch MA, Karlstrom E, Wendel M, Lind T: ADAMTS-1 increases the three-dimensional growth of osteoblasts through type I collagen processing. Bone 2007, 41:231-238.

25. Cabodi S, Taverna D: Interfering with inflammation: a new strategy to block breast cancer self-renewal and progression? Breast Cancer Res 2010, 12:305.

26. Entschladen F, Drell TL, Lang K, Joseph J, Zaenker KS: Tumour-cell migration, invasion, and metastasis: navigation by neurotransmitters. Lancet Oncol 2004, 5:254-258. 
27. Kraljevic Pavelic S, Sedic M, Bosnjak H, Spaventi S, Pavelic K: Metastasis: new perspectives on an old problem. Mol Cancer 2011, 10:22.

28. Hayot C, Debeir O, Van Ham P, Van Damme M, Kiss R, Decaestecker C: Characterization of the activities of actin-affecting drugs on tumor cell migration. Toxicol Appl Pharmacol 2006, 211:30-40.

29. Krampert M, Kuenzle S, Thai SN, Lee N, Iruela-Arispe ML, Werner S: ADAMTS1 proteinase is up-regulated in wounded skin and regulates migration of fibroblasts and endothelial cells. J Biol Chem 2005, 280:23844-23852.

30. Artym W, Zhang Y, Seillier-Moiseiwitsch F, Yamada KM, Mueller SC: Dynamic interactions of cortactin and membrane type 1 matrix metalloproteinase at invadopodia: defining the stages of invadopodia formation and function. Cancer Res 2006, 66:3034-3043.

31. Bowden ET, Onikoyi E, Slack R, Myoui A, Yoneda T, Yamada KM, Mueller SC: Co-localization of cortactin and phosphotyrosine identifies active invadopodia in human breast cancer cells. Exp Cell Res 2006, 312:1240-1253.

32. Kelly T, Yan Y, Osborne RL, Athota AB, Rozypal TL, Colclasure JC, Chu WS: Proteolysis of extracellular matrix by invadopodia facilitates human breast cancer cell invasion and is mediated by matrix metalloproteinases. Clin Exp Metastasis 1998, 16:501-512

33. Mader CC, Oser M, Magalhaes MA, Bravo-Cordero JJ, Condeelis J, Koleske AJ, Gil-Henn H: An EGFR-Src-Arg-cortactin pathway mediates functional maturation of invadopodia and breast cancer cell invasion. Cancer Res 2011, 71:1730-1741.

34. Nascimento CF, Gama-De-Souza LN, Freitas VM, Jaeger RG: Role of MMP9 on invadopodia formation in cells from adenoid cystic carcinoma. Study by laser scanning confocal microscopy. Microsc Res Tech 2010, 73:99-108.

35. Buccione R, Orth JD, McNiven MA: Foot and mouth: podosomes, invadopodia and circular dorsal ruffles. Nat Rev Mol Cell Biol 2004, 5:647-657.

36. Linder S: The matrix corroded: podosomes and invadopodia in extracellular matrix degradation. Trends Cell Biol 2007, 17:107-117.

37. Stylli SS, Kaye AH, Lock P: Invadopodia: at the cutting edge of tumour invasion. J Clin Neurosci 2008, 15:725-737.

38. Chomczynski P, Sacchi N: Single-step method of RNA isolation by acid guanidinium thiocyanate-phenol-chloroform extraction. Anal Biochem 1987, 162:156-159.

doi:10.1186/1476-4598-12-2

Cite this article as: Freitas et al:: Decreased expression of ADAMTS-1 in human breast tumors stimulates migration and invasion. Molecular Cancer 2013 12:2.

\section{Submit your next manuscript to BioMed Central and take full advantage of:}

- Convenient online submission

- Thorough peer review

- No space constraints or color figure charges

- Immediate publication on acceptance

- Inclusion in PubMed, CAS, Scopus and Google Scholar

- Research which is freely available for redistribution 